\title{
Potential Antitumor Effect of Harmine in the Treatment of Thyroid Cancer
}

\author{
Shu Ruan, ${ }^{1,2}$ Feng Jia, ${ }^{3}$ and Jianbo $\mathrm{Li}^{1}$ \\ ${ }^{1}$ Department of Endocrinology, The First Affiliated Hospital of Nanjing Medical University, Nanjing, China \\ ${ }^{2}$ Department of Endocrinology, Yancheng Third Hospital, The Affiliated Hospital of Southeast University Medical College, \\ Yancheng, China \\ ${ }^{3}$ Department of Neurosurgery, Yancheng City No. 1 People's Hospital, The Fourth Affiliated Hospital of Nantong Medical College, \\ Yancheng, China \\ Correspondence should be addressed to Jianbo Li; ljbzjlx18@aliyun.com
}

Received 14 November 2016; Revised 6 January 2017; Accepted 16 January 2017; Published 8 February 2017

Academic Editor: Michael Silbermann

Copyright (C) 2017 Shu Ruan et al. This is an open access article distributed under the Creative Commons Attribution License, which permits unrestricted use, distribution, and reproduction in any medium, provided the original work is properly cited.

\begin{abstract}
Thyroid cancer is one of the most common types of cancer in endocrine system. In latest studies, harmine has been proved to inhibit the growth of several cancers in vitro and in vivo. In the current study, we evaluated the in vitro and in vivo anticancer efficiency of harmine against thyroid cancer cell line TPC-1. The in vitro cytotoxicity of harmine evaluated by XTT assay indicated that harmine suppressed the proliferation of TPC-1 cells in a dose- and time-dependent manner. Moreover, harmine dose-dependently induced apoptosis of TPC- 1 cells through regulating the ratio of Bcl-2/Bax. The colony forming ability of TPC- 1 cells was also timedependently inhibited by harmine. The inhibitory effects of harmine on migration and invasion of TPC-1 cells were studied by wound scratching and Transwell assays. In vivo evaluation showed that harmine effectively inhibited the growth of thyroid cancer in a dose-dependent manner in nude mice. Therefore, harmine might be a promising herbal medicine in the therapy of thyroid cancer and further efforts are needed to explore this therapeutic strategy.
\end{abstract}

\section{Introduction}

Thyroid cancer is one of the most common kinds of cancer in endocrine system which includes four major types with papillary thyroid cancer (PTC) being the most common one [1]. Though most of PTCs respond well to surgery and chemoradiotherapy, part of them quickly develop resistance to chemotherapy, which generate an urgent demand of novel strategy to improve the efficacy of chemotherapeutics [2].

Recently a growing body of evidences demonstrated the promising antitumor efficiency of herbal medicine [3-5]. Several herbal medicines, such as harmine, resveratrol, and berbamine, have shown substantial tumor inhibitory effect against a series of cancers [6-8]. Among all these herbal medicines, harmine, originally isolated from the seeds of Peganum harmala, is a tricyclic compound belonging to the $\beta$-carboline alkaloids [9]. It is reported that harmine possesses various pharmacological activities [10]. In latest studies, harmine has been proved to inhibit the growth of several cancers in vitro and in vivo [11, 12]. For example, harmine induces the apoptosis of cancer cells by leading to DNA break as demonstrated in DNA fragmentation and tunnel study in melanoma cells [13]. Possible mechanisms include the block of homologous recombination, one of the major Double Strand Break (DSB) repair pathways and the inhibition of telomerase activity $[9,12]$. However, no related reports were found on the antitumor effect of harmine in thyroid cancer.

In the current study, we evaluated the in vitro and in vivo anticancer efficiency of harmine against thyroid cancer. The cell inhibition effect of harmine was measured by XTT assay. Apoptosis of thyroid cancer cell line TPC-1 was measured by DAPI staining. Related apoptotic protein expression was evaluated by western blotting. Influences of harmine on the migration and invasion of TPC-1 cells were also examined by wound scratching and Transwell assays. The in vivo antitumor effect was examined in a xenograft model of thyroid cancer. 


\section{Materials and Methods}

2.1. Materials. Harmine was purchased from Sigma Co., Ltd. (St. Louis, USA). Harmine was dissolved in DMSO to make a stock solution at a concentration of $10 \mathrm{mg} / \mathrm{mL}$. Working solution was prepared by diluting the stock solution into medium with the final concentration of DMSO below $0.1 \%$. Human papillary thyroid cancer cell line TPC-1 was purchased from the Institute of Biochemistry and Cell Biology, Chinese Academy of Sciences (Shanghai, China). TPC-1 cells were cultured in RPMI 1640 (w L-glutamate) completed by fetal bovine serum (10\%) and penicillin/streptomycin (1\%) at $37^{\circ} \mathrm{C}$ in a humidified atmosphere containing $5 \% \mathrm{CO}_{2}$.

Male and female nude mice (nu/nu; 6-8 weeks old and weighing 18-22 g) were purchased from the Animal Center of Nanjing Medical University (Nanjing, China). The animal protocol was reviewed and approved by the Institutional Animal Care and Use Committee of Nanjing Medical University.

2.2. In Vitro Cytotoxicity. TPC-1 cells were seeded in 96-well plates with a density of 10000 cells per well and allowed to grow $24 \mathrm{~h}$ before treatment. Cells were then incubated with escalated doses of harmine $(2,4,8,16,32$, and $64 \mu \mathrm{g} / \mathrm{mL})$ for 24,36 , and $48 \mathrm{~h}$. The medium was replaced with $50 \mu \mathrm{L}$ XTT working solution and incubated for another $18 \mathrm{~h}$. The optical density of each well was measured on a microplate reader at $560 \mathrm{~nm}$ (Bio-Rad, Hercules, USA).

2.3. DAPI Staining. Cells were seeded on cover slips in a 24 -well plate and allowed to grow $24 \mathrm{~h}$. Cells were then exposed to three kinds of concentration of harmine $(4,8$, and $16 \mu \mathrm{g} / \mathrm{mL}$ ) for $36 \mathrm{~h}$. After being washed by PBS, cells were fixed by a mixture of cold methanol and acetone $(1: 1)$ for $5 \mathrm{~min}$. $4 \mu \mathrm{g} / \mathrm{mL} 4^{\prime}, 6$-diamidine- $2^{\prime}$-phenylindole dihydrochloride (DAPI) was added to the well and allowed to incubate for $10 \mathrm{~min}$ at room temperature. Cells were imaged under a fluorescent microscope.

2.4. Western Blot. TPC-1 cells were incubated with harmine (4, 8, and $16 \mu \mathrm{g} / \mathrm{mL})$ for $6 \mathrm{hrs}$. Cell total proteins were extracted and quantified according to the instruction of the kit. $50 \mu \mathrm{g}$ of proteins was loaded into each well during the electrophoresis. Protein expression was measured by western blot as reported previously. Primary antibodies (anti-Bax, anti-Bcl-2, and anti- $\beta$-actin) were purchased from Sigma Co., Ltd. (St. Louis, MO, USA).

2.5. Clonogenic Assay. The first clonogenic assay was performed to study the inhibition of harmine on the colony forming ability of TPC-1 cells. Cells were treated with harmine $(4,8$, and $16 \mu \mathrm{g} / \mathrm{mL})$ for 24,36 , and $48 \mathrm{~h}$. Another clonogenic assay was performed to evaluate the radiosensitizing effect of harmine. TPC-1 cells were treated with $2 \mu \mathrm{g} / \mathrm{mL}$ harmine and escalated doses of radiation singly or in combination. After each treatment, cells were trypsinized, collected, and set to the calculated concentration. After 10-14 days of incubation in 6-well plates, the 6-well plates were dyed by $0.5 \%$ crystal violet and counted as reported previously [14].
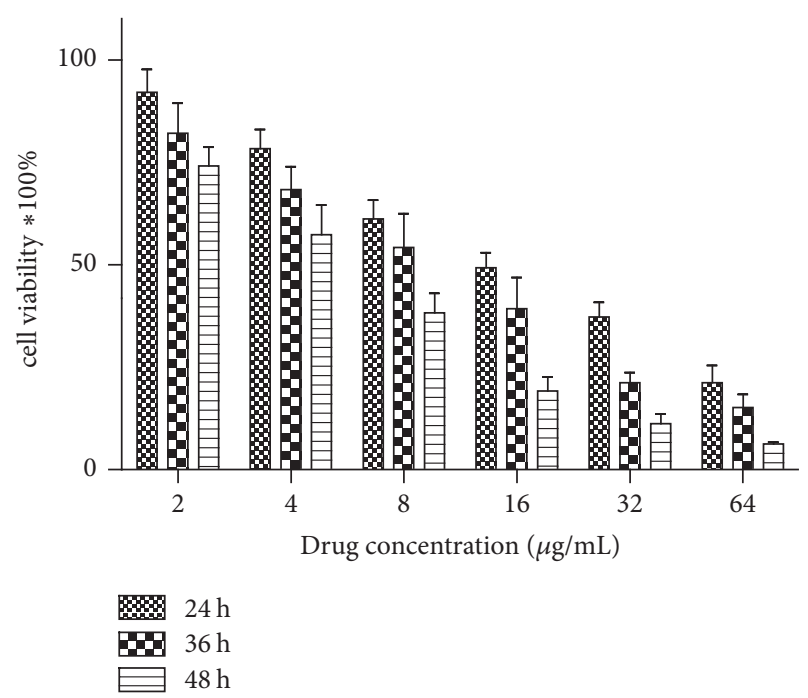

FIgURE 1: In vitro cytotoxicity of harmine on TPC-1 cells for the incubation of 24,36 , and $48 \mathrm{hrs}$.

2.6. DNA Fragmentation Assay. TPC-1 cells were seeded in a 24 -well plate and allowed to grow for $24 \mathrm{~h}$. Cells were then exposed to three kinds of concentration of harmine $(4,8$, and $16 \mu \mathrm{g} / \mathrm{mL}$ ) for $36 \mathrm{~h}$. Total DNA was extracted and run by gel electrophoresis as previously reported [15].

2.7. Wound Scratching Assay and Transwell Assay. Cells were seeded in 6-well plate and allowed to grow for $24 \mathrm{~h}$ to form a monolayer. Then a tip was utilized to scratch in the middle to generate a clear space (wound). Immediately it is captured by the camera and was designated as $0 \mathrm{~h}$. Then the medium was replaced with serum-free medium containing different concentrations of harmine $(4,8$, and $16 \mu \mathrm{g} / \mathrm{mL})$. After $36 \mathrm{~h}$, another image was captured to measure the width of the wound. The wound healing rate was calculated by the following formula: the average width of wound at $0 \mathrm{~h}-$ the average width of wound at $36 \mathrm{~h} /$ the average width of wound at $0 \mathrm{~h}$.

Transwell assays were performed in 24-well Transwell chambers (Corning, Acton, MA, USA) as reported previously. Briefly, TPC-1 cells were seeded in the upper chamber in serum-free medium, while the bottom chamber was filled with complete medium as an attractant. $36 \mathrm{~h}$ later, the successfully invaded cells on the bottom chamber were stained by crystal violet, counted, and plotted.

2.8. In Vivo Antitumor Efficacy. Briefly, 1.5 million TPC-1 cells were injected subcutaneously in the left axillary space of the mice. 10 days later the mice with the tumor nodule reaching a volume of $70-80 \mathrm{~mm}^{3}$ were randomly divided into four groups with 6 mice per group. Escalated doses of harmine were administered by tail vein injection.

The mice were then treated with escalated doses of harmine with saline as the control. Tumor volume was measured by a caliper every other day during the whole experiment. The tumor volume (TV) was calculated with the 


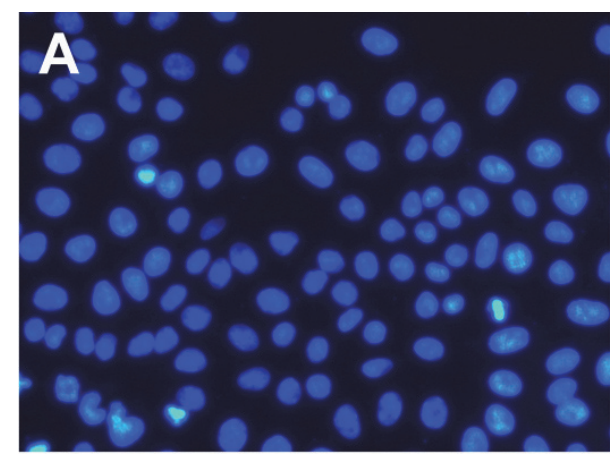

(a)

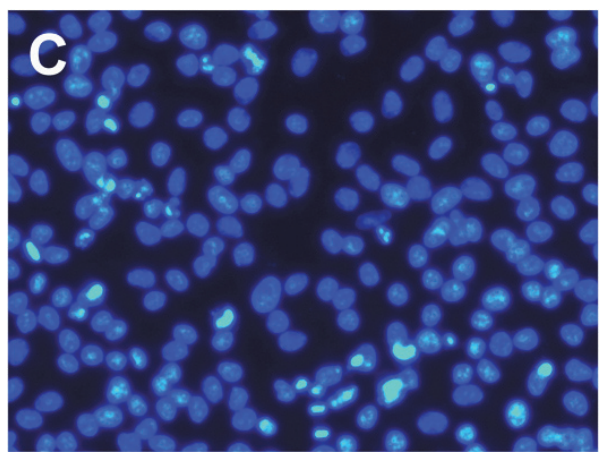

(c)

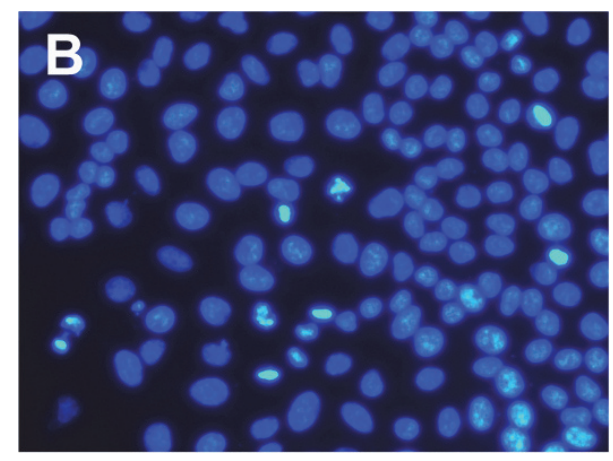

(b)

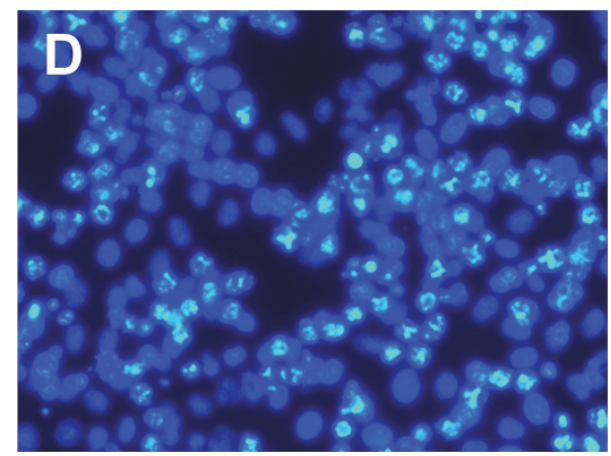

(d)

FIgURE 2: Apoptosis of TPC-1 cells detected by DAPI staining. (a) The nontreated cells. (b) Cells were treated with $4 \mu \mathrm{g} / \mathrm{mL}$ harmine for $36 \mathrm{~h}$. (c) Cells were treated with $8 \mu \mathrm{g} / \mathrm{mL}$ harmine for $36 \mathrm{~h}$. (d) Cells were treated with $16 \mu \mathrm{g} / \mathrm{mL}$ harmine for $36 \mathrm{~h}$.

following formula: TV $=\left(W^{2} * L\right) / 2$, where $W$ is the width of tumor nodule and $L$ is the length of tumor nodule.

2.9. Statistical Analysis. Results were presented as mean \pm SD. Statistical analysis was made by Student's $t$-test or ANOVA. The $p$ value $<0.05$ was considered as significant.

\section{Results}

3.1. Cytotoxicity and Apoptotic Induction of Harmine on TPC1 Cells. As shown in Figure 1, escalated doses of harmine effectively led to the decrease of cell viability at different incubation times (Figure 1). The IC50 values of harmine against TPC- 1 cells at 24, 36, and $48 \mathrm{~h}$ were $16.57 \pm 1.4$, $9.48 \pm 1.1$, and $5.51 \pm 0.7 \mu \mathrm{g} / \mathrm{mL}$, respectively. It is noted that the IC50 values significantly lowered as the incubation time extended. Therefore, harmine inhibited the proliferation of TPC-1 cells in a time- and dose-dependent manner.

As shown in Figure 2, it is obvious to locate the characterized morphology of apoptotic cells in harmine treated group with brighter DAPI staining with condensed chromatin forming crescent-shaped profiles around the periphery of the nucleus or separate globular structures (apoptotic bodies). Quantitative analysis indicated the significant differences among the three groups treated with elevated doses of harmine, which was in accordance with the results in cytotoxicity tests (Figure 3 ). Figure 4 indicated that harmine was effective to induce the fragmentation of DNA of TPC-1 cells

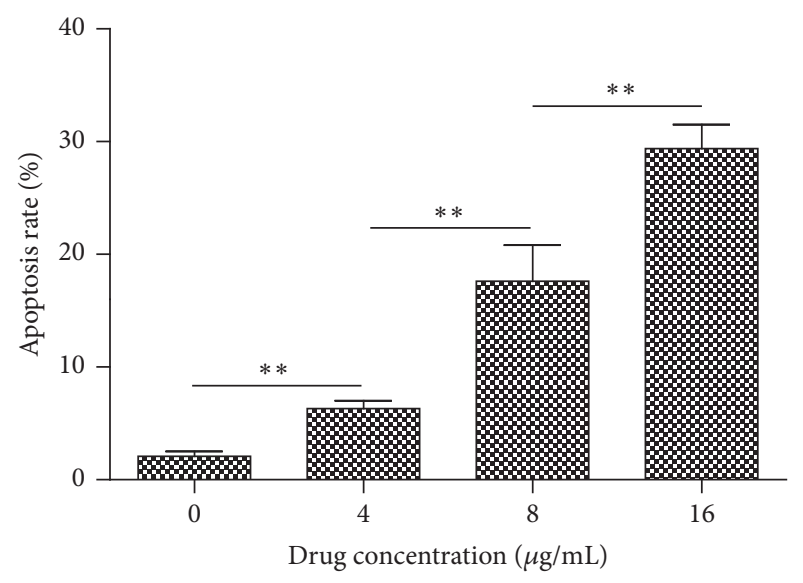

FIGURE 3: Quantitative analysis of apoptotic rate of cells exposed to different agents. Values are represented as mean $\pm \operatorname{SD}(n=3) . * *$ means $p<0.01$.

in a dose-dependent manner, demonstrating the apoptosis inducing effect.

3.2. Effect of Harmine on Apoptotic Proteins and the Activity of Caspase-3. To examine the possible mechanism of apoptotic induction of harmine, the expression of apoptosis related proteins was evaluated by western blot. As shown in Figure 5, treatment of harmine dose-dependently induced the expression of Bax and degraded the existing Bcl-2, leading 


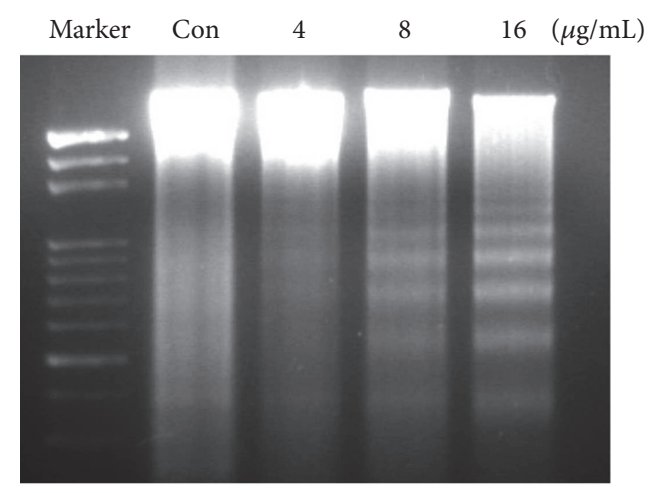

FIgURE 4: DNA fragmentation assay of TPC-1 cell treated with escalated doses of harmine. Cells were treated with different concentrations of harmine $(4,8$, and $16 \mu \mathrm{g} / \mathrm{mL})$ for $36 \mathrm{~h}$ and then total DNA was extracted and electrophoresed.

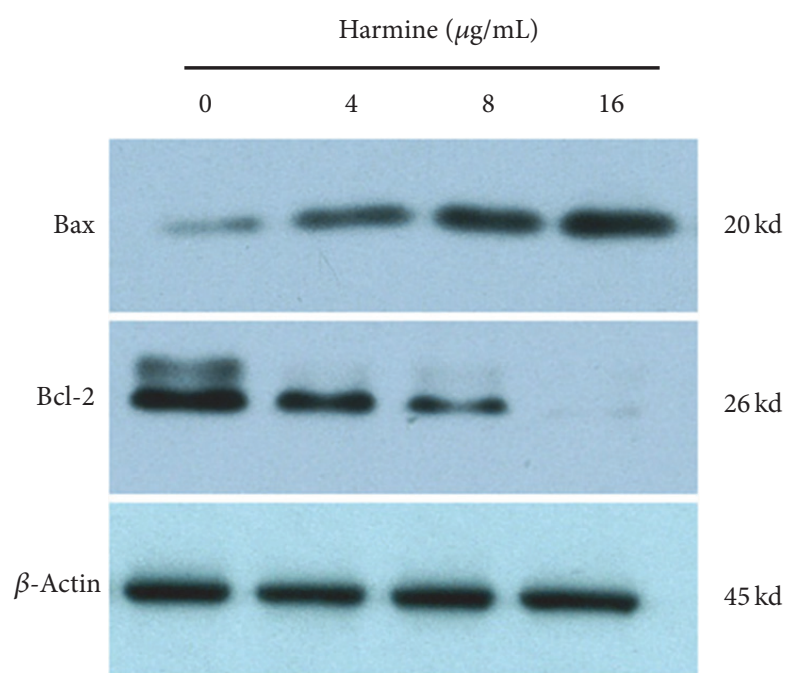

FIGURE 5: Protein expression of TPC-1 cells treated with different doses of harmine.

to a significant decrease in the ratio of $\mathrm{Bcl}-2 / \mathrm{Bax}$, which demonstrated the progression of apoptosis.

The activity of Caspase- 3 in TPC- 1 cells treated with a series of doses of harmine was evaluated by the caspase colorimetric protease assay kit. As one of the crucial mediators of apoptosis, Caspase- 3 is considered as an apoptotic marker due to its significant role in catalyzing the specific cleavage of key proteins and generation of apoptotic bodies. Figure 6 showed that harmine was effective in elevating the activity of Caspase-3, which was in accordance with the results of apoptosis detection from Figures 2 and 3.

3.3. Effect of Harmine on the Colony Forming Ability of TPC-1 Cells with or without the Combination of Radiation. Figure 7(a) showed the clonogenic assay of harmine on TPC-1 cells. The colony forming ability of TPC- 1 cells was time- and dose-dependently inhibited by harmine. The percent survival of TPC-1 cells decreased as the concentration of harmine increased and incubation time extended, demonstrating that

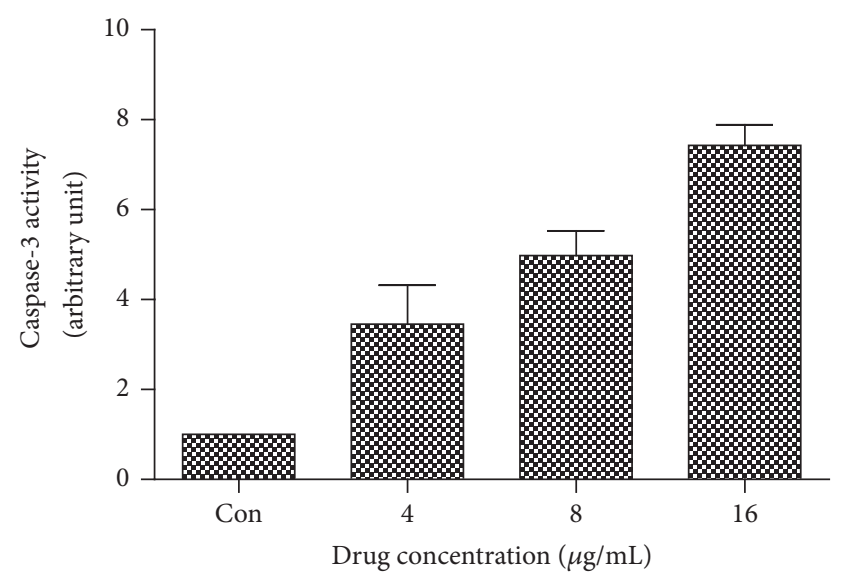

FIgUre 6: The activity of Caspase-3 in TPC-1 cells treated with different doses of harmine.

the antiproliferative effect of harmine was consistent with the results from cytotoxicity test.

Figure $7(\mathrm{~b})$ indicated that $2 \mu \mathrm{g} / \mathrm{mL}$ harmine effectively enhanced the inhibitory effect of radiation on the colony forming ability of TPC- 1 cells. There was a significant difference between the percent survival of radiation alone and radiation plus harmine, which demonstrated the potential radiosensitizing effect of harmine.

\subsection{Effects of Harmine on the Migration and Invasion of} TPC-1 Cells. Figure 8 indicated the effect of harmine on the wound healing rate of TPC-1 cells. Harmine dosedependently decreased the wound healing rate of TPC-1 cells, which demonstrated an inhibitory effect of harmine on the migration of thyroid cancer cells. Similarly, harmine inhibited the invasion of TPC-1 cells in a dose-dependent manner as shown in Figure 9. Statistical analysis indicated significant differences among the groups treated with different doses of harmine. Similarly, the number of invading cells decreased significantly as the concentration of harmine increased. Results from wound scratch and Transwell assays demonstrate that harmine is effective in restraining the mobility of TPC-1 cells in a dose-dependent manner, which makes it a potential drug for the reverse of tumor metastasis.

3.5. In Vivo Antitumor Evaluation of Harmine against TPC1 Xenograft. In vivo antitumor effects of harmine were examined in a xenograft model of TPC-1 cells in nude mice (Figure 10). It is obvious that harmine delayed the growth of thyroid cancer in a dose-dependent manner. However, though the tumor of the mice receiving the lowest dose of harmine grew more slowly than that of the mice in the control group, there was no significant difference between the two groups. On the contrary, the other two relatively high doses of harmine significantly inhibit the growth of tumor when compared with the control group. Among all the three groups, the highest dose of harmine generated the greatest antitumor effect. Moreover, $40 \mathrm{mg} / \mathrm{kg}$ harmine restrained the tumor growth more significantly than $20 \mathrm{mg} / \mathrm{kg}$ harmine. In 


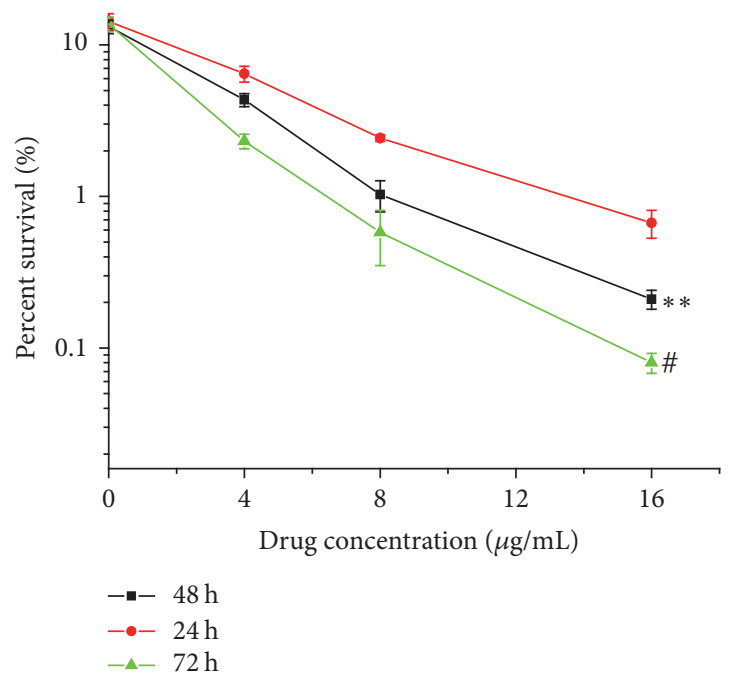

(a)

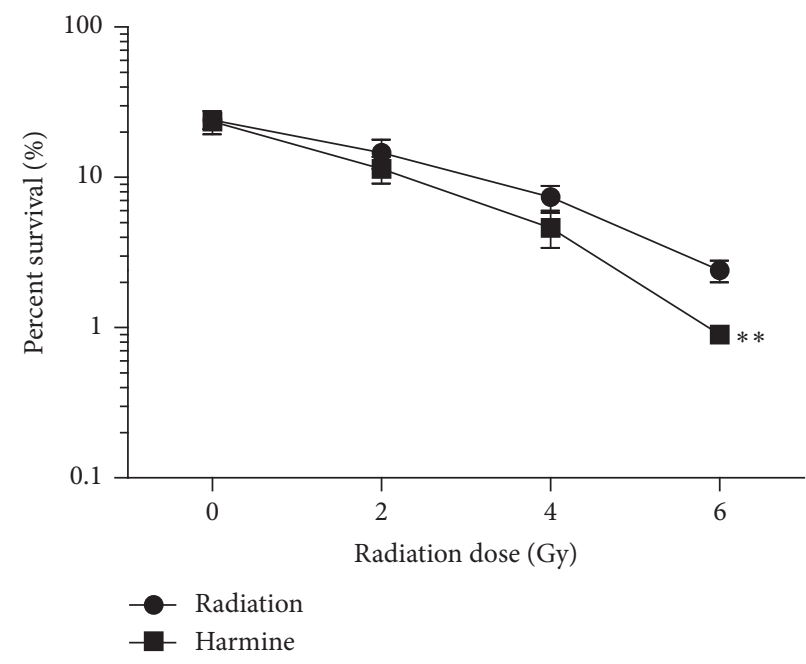

(b)

FIGURE 7: Clonogenic assay of TPC-1 cells treated with harmine and radiation. (a) Inhibitory effect of different doses of harmine on the colony forming ability of TPC- 1 cells. $* *$ represents $p<0.01$ versus $24 \mathrm{~h}$ group. \# represents $p<0.01$ versus $48 \mathrm{~h}$ group. (b) Radiosensitization effect of harmine $(2 \mu \mathrm{g} / \mathrm{mL})$ on TPC- 1 cells. $* *$ represents $p<0.01$ versus radiation group.

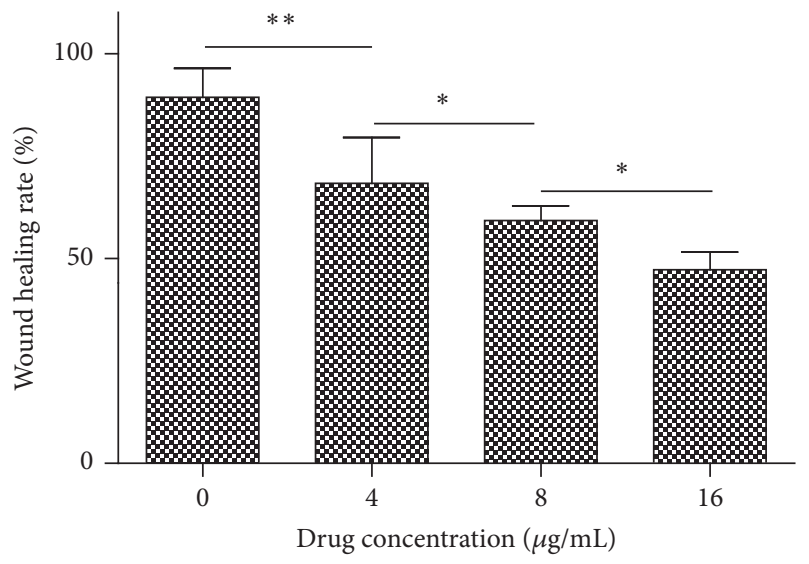

FIGURE 8: Wound healing ability of TPC-1 cells exposed to a series of doses of harmine. Quantification of cell migration using the monolayer wound scratching assay. $*$ represents $p<0.05$. ** represents $p<0.01$.

addition, there was no obvious toxicity (such as weight loss or behavior) in all the mice during the whole experiment.

\section{Discussion}

Here we show the cytotoxic and antiproliferative effect of harmine on the in vitro and in vivo model of thyroid cancer. As reported in previous studies, harmine showed cytotoxic and antiproliferative effect against several kinds of cancers [11, 16]. Several intracellular targets have been identified such as cyclin proteins and interference with DNA [17]. However, no report was about the antitumor effect of harmine in thyroid cancer.

In the current study, we demonstrated a time- and dosedependent cytotoxicity of harmine against thyroid cancer cell

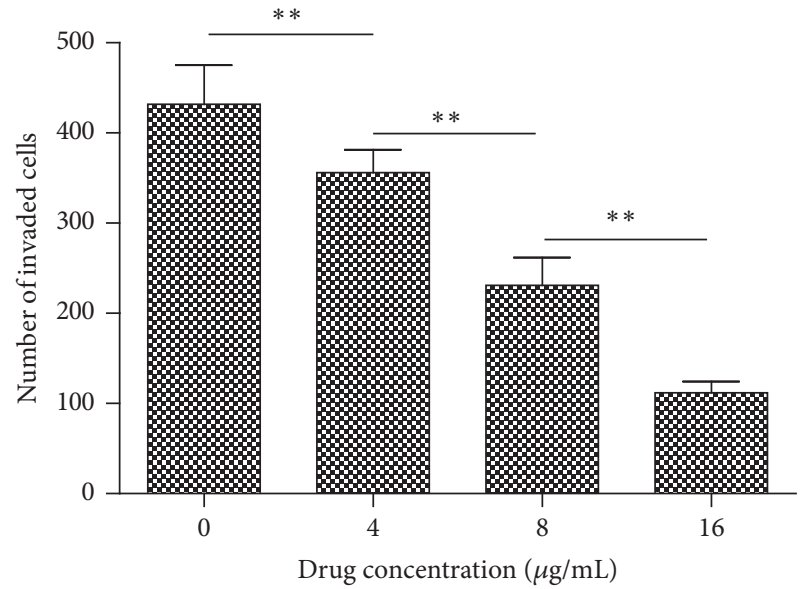

FIGURE 9: Cell invasive ability of TPC-1 cells exposed to harmine. Quantification of the numbers of invaded cells exposed to a series of doses of harmine. Each data point represents the mean \pm SD from three independent experiments. $* *$ represents $p<0.01$.

line TPC-1. Both XTT and clonogenic assay showed that the antiproliferative effect of harmine was mainly dependent on the concentration and incubation time of harmine. Several studies have reported the dose-dependent cytotoxicity of harmine against other kinds of cancers. For example, Zhang et al. reported the time- and dose-dependent antiproliferative effect of harmine against two kinds of gastric cancer cells [11]. A recent study from South China also demonstrated the inhibitory effect of harmine derivative on the proliferation of gastric cancer cells [7].

Though most of the studies focused on the antiproliferative effect of harmine, few researchers evaluated the influence of harmine on the colony forming ability of cancer cells as 


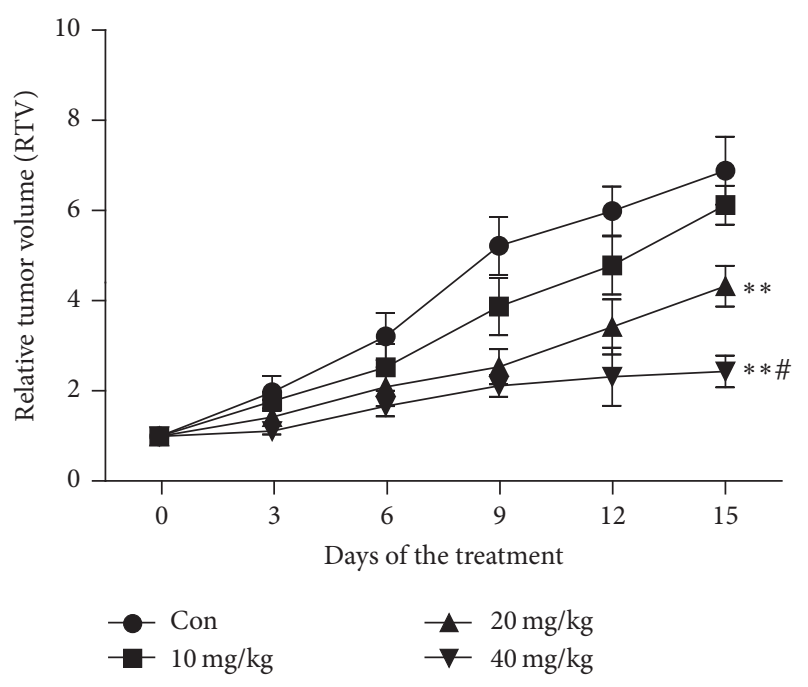

FIGURE 10: Antitumor effect of harmine in TPC-1 xenograft models. Tumor volume of established TPC-1 xenografts in nude mice during therapy under different treatments. Mice were treated with different protocols on Day 0 as shown in the figure. Saline: vehicle; harmine was administered at the doses of 10,20 , and $40 \mathrm{mg} / \mathrm{kg}$. Different agents were delivered through intravenous pathway when tumor volume measured $100 \mathrm{~mm}^{3}$. Data are presented as mean $\pm \mathrm{SD}(n=$ 6 ). The difference between tumor volumes in the group of saline and either 20 or $40 \mathrm{mg} / \mathrm{kg}$ of harmine is significant ( $* *$ means $p<0.01$ versus control). Significant difference (\# means $p<0.05$ ) also is observed between the groups receiving $40 \mathrm{mg} / \mathrm{kg}$ and $20 \mathrm{mg} / \mathrm{kg}$ harmine.

well as the radiosensitizing effect. As introduced previously, loss of reproductive integrity and the inability to proliferate indefinitely is a key feature to cell death $[14,18]$. Cells with integrity but unable to divide and produce a large number of colony are considered dead [19]. Therefore, cells with both the integrity and the ability to proliferate are referred to as "clonogenic," which is pivotal to the proliferation of cancer cells and regarded as a marker of cancer malignancy [20]. Here we showed that harmine exerted a time- and dosedependent inhibition on the clonogenic ability of TPC-1 cells, which means that harmine could effectively disrupt the reproductive integrity and lead to the inability of proliferation of thyroid cancer cells. Moreover, the current study reported for the first time that harmine showed the potential to be a promising radiosensitizer.

The following DAPI staining of cancer cells indicated that harmine was efficient to induce apoptosis of TPC-1 cells. It is known that apoptosis, also called programmed cell death, is characterized by the specific morphology, such as chromatin condensation, membrane blebbing, internucleosome degradation of DNA, and apoptotic body formation [21]. It is obvious that cells treated with harmine underwent some characteristic phenomenon of apoptosis. It is reported in earlier studies that the Bcl-2 family, including proapoptotic proteins and antiapoptotic proteins, plays an important role in apoptosis $[22,23]$. Among all these proteins, the ratio of Bcl-2 and Bax is a key indicator of apoptosis [24]. Figure 4 showed that the treatment by harmine dose-dependently decreased the expression of Bcl-2 while simultaneously increased the expression of Bax, which led to an obvious decline in the ratio of Bcl-2 and Bax. Though the effect of apoptotic induction by harmine was also reported by other groups, different mechanisms underlying the antitumor effect of harmine have been identified such as cell cycle arrest and telomerase activity inhibition [9]. The anticancer effect of harmine was confirmed in TPC-1 xenograft model, which, together with other studies focusing on harmine, proved the efficacy of harmine in the treatment of cancer.

The ability of migration and invasion is the characteristic of malignancy, which often leads to the failure of cancer therapy [25]. The efficient inhibition of tumor migration and invasion is an effective way to control the metastasis of tumor. Since few studies examined the antimetastatic effect of harmine, we evaluate the ability of harmine on cell migration and invasion through wound scratching and Transwell assays. Results from the two experiments demonstrated the dosedependent inhibitory effect of harmine in vitro. Moreover, animal experiment on metastatic xenograft models in the author's lab is ongoing to further evaluate the in vivo antimetastasis efficacy of harmine.

As mentioned above, evidences from our report not only demonstrate the potential antitumor effect of harmine, but also provide a feasible way to counteract the metastasis of thyroid cancer. Moreover, further studies focusing on the chemosensitization effect of harmine are ongoing in the author's lab to explore more potential application of such alkaloids. It is undoubtedly, however, that the development of herbal medicine, especially alkaloids, warrants more intensive research in order to evaluate the feasibility and advantages of clinical applications.

In summary, the current study demonstrates the antitumor effect of harmine in the treatment of thyroid cancer. In vitro cytotoxicity tests indicate that harmine possesses a doseand time-dependent cell inhibitory and apoptosis inducing effect on TPC-1 cells. In addition, harmine effectively inhibits the migration and invasion of TPC-1 cells in a dosedependent manner. Moreover, in vivo evaluation shows that harmine significantly delays the growth of thyroid cancer in a dose-dependent manner in nude mice. Therefore, harmine might be a promising herbal medicine in tumor therapy and further efforts are needed to explore this therapeutic strategy.

\section{Competing Interests}

The authors report no conflict of interests in this work.

\section{References}

[1] M. E. Cabanillas, D. G. McFadden, and C. Durante, “Thyroid cancer," The Lancet, vol. 388, no. 10061, pp. 2783-2795, 2016.

[2] E. Tirrò, M. L. Consoli, M. Massimino et al., "Altered expression of c-IAP1, survivin, and smac contributes to chemotherapy resistance in thyroid cancer cells," Cancer Research, vol. 66, no. 8, pp. 4263-4272, 2006.

[3] X. Wei, Z. Chen, X. Yang, and T. Wu, "Chinese herbal medicines for esophageal cancer," Cochrane Database of Systematic Reviews, no. 4, Article ID CD004520, 2009. 
[4] L. Huang, J. Zhang, T. Song et al., "Antifungal curcumin promotes chitin accumulation associated with decreased virulence of Sporothrix schenckii," International Immunopharmacology, vol. 34, pp. 263-270, 2016.

[5] Y. Hu, S. Wang, X. Wu et al., "Chinese herbal medicine-derived compounds for cancer therapy: a focus on hepatocellular carcinoma," Journal of Ethnopharmacology, vol. 149, no. 3, pp. 601-612, 2013.

[6] L. Tan, W. Wang, G. He et al., "Resveratrol inhibits ovarian tumor growth in an in vivo mouse model," Cancer, vol. 122, no. 5, pp. 722-729, 2016.

[7] P. Zhang, C.-R. Huang, W. Wang et al., "Harmine hydrochloride triggers G2 phase arrest and apoptosis in MGC-803 cells and SMMC-7721 cells by upregulating p21, activating caspase-8/Bid, and downregulating ERK/bad pathway," Phytotherapy Research, vol. 30, no. 1, pp. 31-40, 2016.

[8] T. Liu, X. Liu, and W. Li, "Tetrandrine, a Chinese plant-derived alkaloid, is a potential candidate for cancer chemotherapy," Oncotarget, vol. 7, no. 26, pp. 40800-40815, 2016.

[9] L. Zhao and M. Wink, "The $\beta$-carboline alkaloid harmine inhibits telomerase activity of MCF-7 cells by down-regulating hTERT mRNA expression accompanied by an accelerated senescent phenotype," PeerJ, vol. 1, article e174, 2013.

[10] T. P. Hamsa and G. Kuttan, "Harmine inhibits tumour specific neo-vessel formation by regulating VEGF, MMP, TIMP and pro-inflammatory mediators both in vivo and in vitro," European Journal of Pharmacology, vol. 649, no. 1-3, pp. 64-73, 2010.

[11] H. Zhang, K. Sun, J. Ding et al., "Harmine induces apoptosis and inhibits tumor cell proliferation, migration and invasion through down-regulation of cyclooxygenase-2 expression in gastric cancer," Phytomedicine, vol. 21, no. 3, pp. 348-355, 2014.

[12] L. Zhang, F. Zhang, W. Zhang et al., "Harmine suppresses homologous recombination repair and inhibits proliferation of hepatoma cells," Cancer Biology and Therapy, vol. 16, no. 11, pp. 1585-1592, 2015.

[13] T. P. Hamsa and G. Kuttan, "Harmine activates intrinsic and extrinsic pathways of apoptosis in B16F-10 melanoma," Chinese Medicine, vol. 6, no. 1, article 11, 2011.

[14] H. Rafehi, C. Orlowski, G. T. Georgiadis, K. Ververis, A. ElOsta, and T. C. Karagiannis, "Clonogenic assay: adherent cells," Journal of Visualized Experiments, no. 49, 2011.

[15] K. Yuki, A. Takahashi, I. Ota et al., "Sensitization by glycerol for CDDP-therapy against human cultured cancer cells and tumors bearing mutated p53 gene," Apoptosis, vol. 9, no. 6, pp. 853-859, 2004.

[16] H. Liu, D. Han, Y. Liu et al., "Harmine hydrochloride inhibits Akt phosphorylation and depletes the pool of cancer stem-like cells of glioblastoma," Journal of Neuro-Oncology, vol. 112, no. 1, pp. 39-48, 2013.

[17] Y. Song, D. Kesuma, J. Wang et al., "Specific inhibition of cyclin-dependent kinases and cell proliferation by harmine," Biochemical and Biophysical Research Communications, vol. 317, no. 1, pp. 128-132, 2004.

[18] N. A. P. Franken, H. M. Rodermond, J. Stap, J. Haveman, and C. van Bree, "Clonogenic assay of cells in vitro," Nature Protocols, vol. 1, no. 5, pp. 2315-2319, 2006.

[19] A. Munshi, M. Hobbs, and R. E. Meyn, "Clonogenic cell survival assay," Methods in Molecular Medicine, vol. 110, pp. 21-28, 2005.

[20] J. A. Plumb, "Cell sensitivity assays: clonogenic assay," Methods in Molecular Medicine, vol. 88, pp. 159-164, 2004.
[21] S. J. Martin and D. R. Green, "Apoptosis and cancer: the failure of controls on cell death and cell survival," Critical Reviews in Oncology and Hematology, vol. 18, no. 2, pp. 137-153, 1995.

[22] P. Gazzaniga, A. Gradilone, R. Vercillo et al., "bcl-2/bax mRNA expression ratio as prognostic factor in low-grade urinary bladder cancer," International Journal of Cancer, vol. 69, no. 2, pp. 100-104, 1996.

[23] L. F. Gibson, J. Fortney, G. Magro, S. G. Ericson, J. P. Lynch, and K. S. Landreth, "Regulation of BAX and BCL-2 expression in breast cancer cells by chemotherapy," Breast Cancer Research and Treatment, vol. 55, no. 2, pp. 107-117, 1999.

[24] S. Cory and J. M. Adams, "Killing cancer cells by flipping the Bcl-2/Bax switch," Cancer Cell, vol. 8, no. 1, pp. 5-6, 2005.

[25] C. E. Henry, E. Llamosas, A. Djordjevic, N. F. Hacker, and C. E. Ford, "Migration and invasion is inhibited by silencing ROR1 and ROR2 in chemoresistant ovarian cancer," Oncogenesis, vol. 5, no. 5, article no. e226, 2016. 


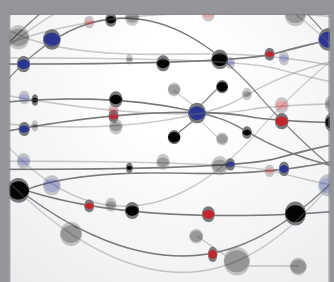

The Scientific World Journal
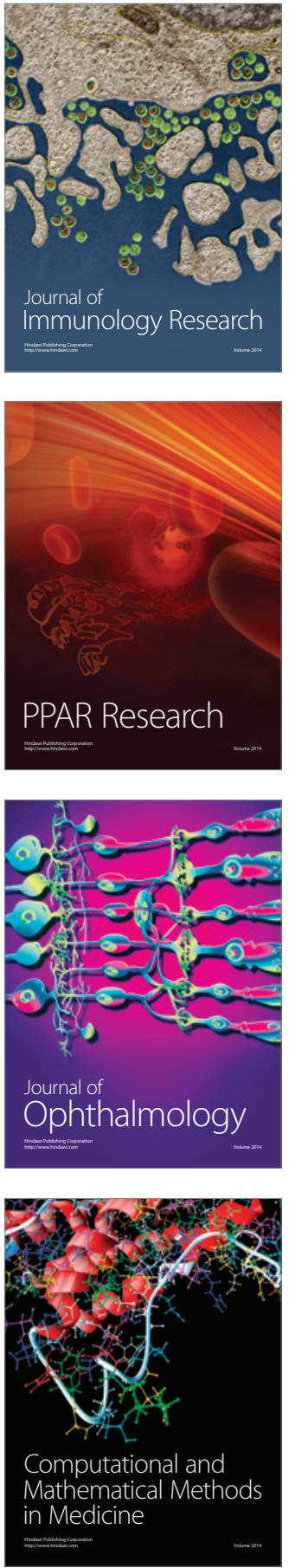

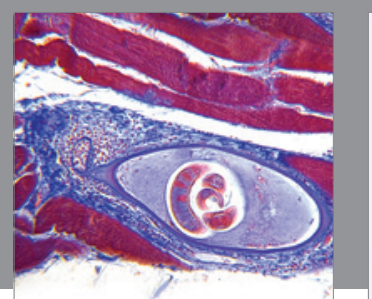

Gastroenterology Research and Practice
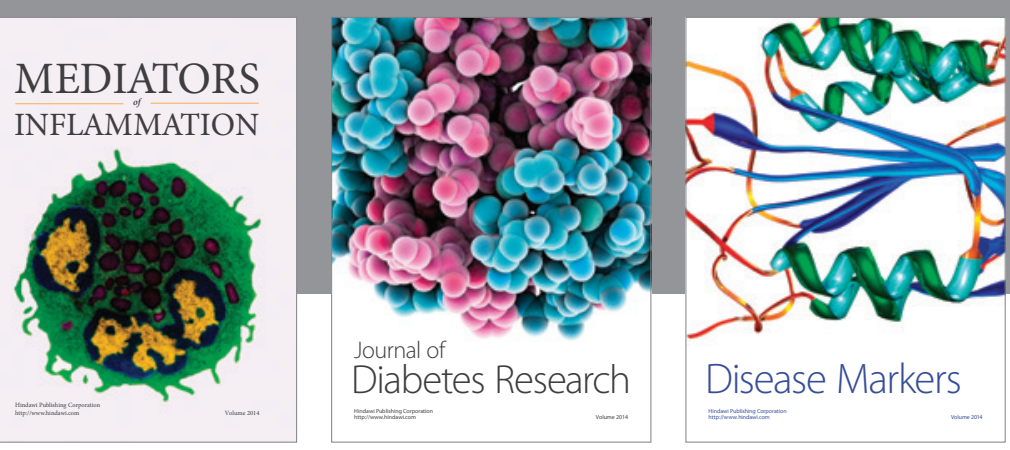

Disease Markers

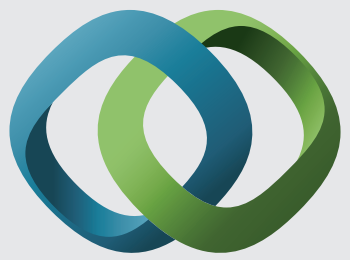

\section{Hindawi}

Submit your manuscripts at

https://www.hindawi.com
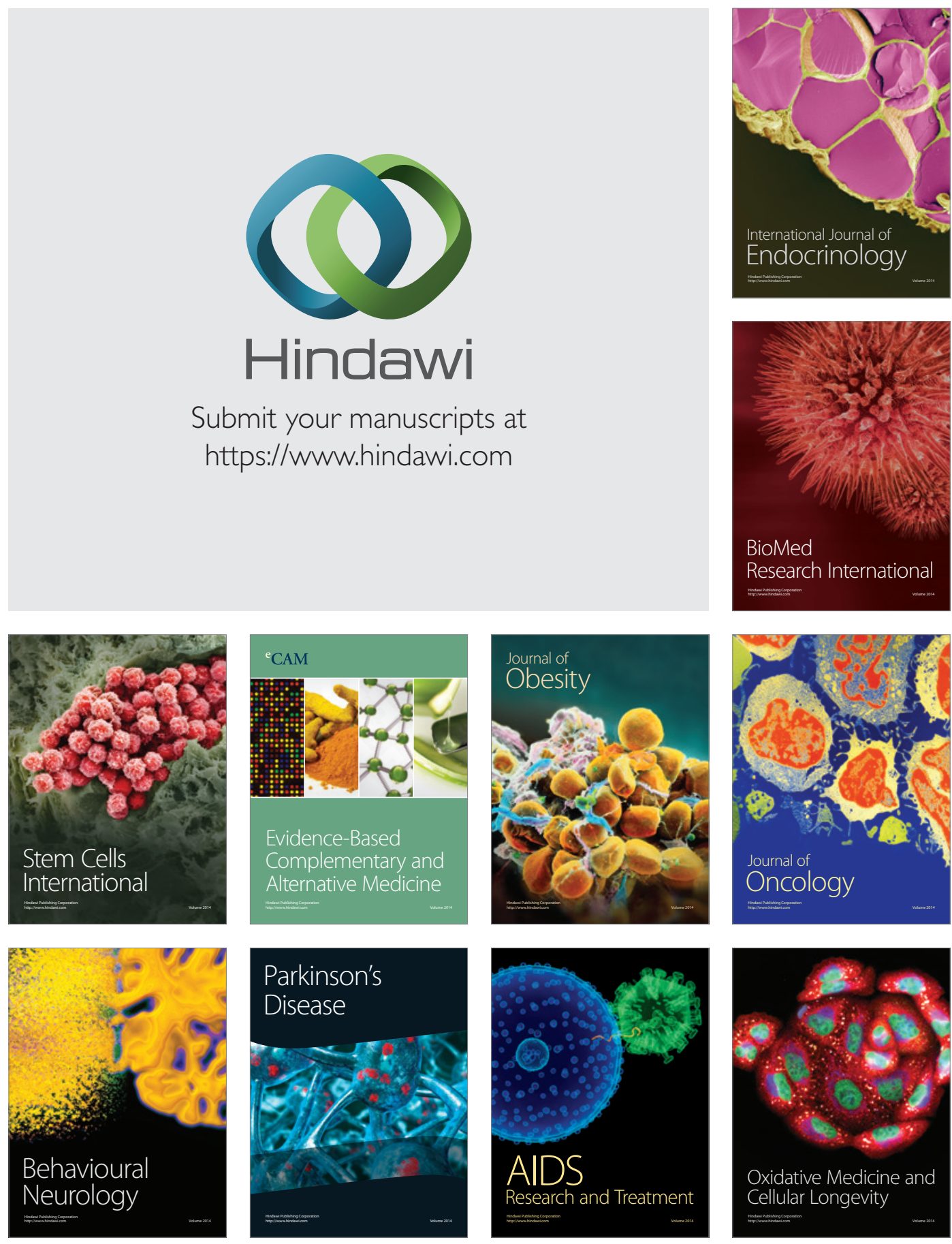